\title{
On the Performance Analysis of Digital Communications over Generalized- $K$ Fading Channels
}

\author{
Petros S. Bithas, Student Member, IEEE, Nikos C. Sagias, Member, IEEE, \\ P. Takis Mathiopoulos, Senior Member, IEEE, George K. Karagiannidis, Senior Member, IEEE, \\ and Athanasios A. Rontogiannis, Member, IEEE
}

\begin{abstract}
The performance of digital communication systems over Generalized- $K\left(K_{G}\right)$ fading channels is analyzed and evaluated. Novel closed form expressions for the SNR statistics, the average Shannon's channel capacity and the bit error rate (BER) are derived. These expressions are used to study important performance criteria such as the outage performance, the average capacity and the BER for a great variety of modulation formats in $K_{G}$ fading channels. The proposed mathematical analysis is accompanied with various performance evaluation results, which demonstrate the usefulness of the proposed approach.
\end{abstract}

Index Terms-Generalized- $K$ distribution, fading and shadowing channels, bit error rate (BER), outage probability, capacity.

\section{INTRODUCTION}

$\mathbf{R}$ ADIO-wave propagation through wireless channels is a complicated phenomenon characterized by various effects including multipath fading and shadowing. Depending upon the nature of the radio propagation environment there are different models describing the statistical behavior of multipath fading [1]. Two relatively new models for fading channels are the $K$ and the Generalized- $K\left(K_{G}\right)$ distributions [2], which in the past have been widely used in radar applications [3], [4]. The $K_{G}$ fading model is quite general as it includes the $K$ distribution as a special case and accurately approximates many other fading models, such as Nakagami- $m$ and Rayleigh-Lognormal (R-L) [2], [5]. Representative past work concerning the $K$ distribution can be found in [5][7]. In [5] it has been shown that the $K$ distribution model provides similar performance to the well-known R-L model with the former being also mathematically more tractable. In [6], the parameters of the $K$ distribution were estimated using a combination of the maximum likelihood principle and the method of moments. Closed form expressions for the bit error rate (BER) of binary differential phase-shift keying (BDPSK) and minimum-shift keying (MSK) signals transmitted over

Manuscript received July 27, 2005. The associate editor coordinating the review of this letter and approving it for publication was Dr. Javier Gozalvez.

P. S. Bithas, N. C. Sagias, P. T. Mathiopoulos, and A. A. Rontogiannis are with the Institute for Space Applications and Remote Sensing, National Observatory of Athens, Metaxa \& Vas. Pavlou Street, Palea Penteli, 15236 Athens, Greece (e-mail: \{pbithas, nsagias, mathio, tronto\}@ space.noa.gr).

G. K. Karagiannidis is with the Department of Electrical and Computer Engineering, Aristotle University of Thessaloniki, 54124 Thessaloniki, Greece (e-mail: geokarag@auth.gr).

Digital Object Identifier 10.1109/LCOMM.2006.05030.
$K$ and R-L fading channels have been derived in [7] also verifying the closed performance agreement between these two fading models.

In the context of digital communications over fading channels, the $K_{G}$ distribution has been used only recently. In [2] it was argued that the $K_{G}$ distribution is general enough to model the fading and shadowing phenomena encounter in mobile communication channels. Furthermore, the main advantage of employing the $K_{G}$ distribution is that it makes mathematical performance analysis much simpler to handle as compared to Lognormal-based models, e.g., the Nakagami$m$ or the R-L model. To justify this observation, in [2], the amount of fading (AF), which is a unified measure of the severity of fading [1], and the BER for the special case of binary phase shift keying (BPSK) modulation have been derived for the $K_{G}$ fading channels. However, a detailed performance analysis of the signal-to-noise ratio (SNR) statistics and the performance of more general receiver structures operating over such channels is not available in the open technical literature, and thus is the topic of our contribution.

\section{The $K_{G}$ FAding Model}

\section{A. Channel Model Statistics}

Let the fading amplitude $X$ be a random variable following the $K_{G}$ distribution. Its probability density function (PDF) is given by [2]

$f_{X}(x)=\frac{4 m^{(\beta+1) / 2} x^{\beta}}{\Gamma(m) \Gamma(k) \Omega^{(\beta+1) / 2}} K_{\alpha}\left[2\left(\frac{m}{\Omega}\right)^{1 / 2} x\right], \quad x \geq 0$

with $\alpha=k-m, \beta=k+m-1$, where $k, m$ are the distribution shaping parameters, $K_{\alpha}(\cdot)$ is the modified Bessel function of order $\alpha$ [8, eq. (8.407/1)], $\Gamma(\cdot)$ is the Gamma function [8, eq. (8.310/1)]. Furthermore, $\Omega$ is the mean power defined as $\Omega \triangleq E\left\langle X^{2}\right\rangle / k$, [2, eq. (7)], with $E\langle\cdot\rangle$ denoting expectation. Since $K_{G}$ is a two parameter distribution, (1) can describe various fading and shadowing models by using different value combinations for $k$ and/or $m$. For example, as $k \rightarrow \infty$, it approximates the well-known Nakagami$m$ distribution [2]; for $m=1$, it coincides with the $K$ distribution and approximately models R-L fading conditions [2], [5]; while for $m \rightarrow \infty$ and $k \rightarrow \infty$, (1) approaches the additive white Gaussian noise (AWGN) channel. 
Considering $K_{G}$ fading channel, the equivalent baseband received signal can be expressed as $z=s X+n$, where $s$ is the transmitted symbol, which can take values from different modulation alphabets, e.g., $M$-phase shift keying (PSK) and $M$-quadrature amplitude modulation (QAM), and $n$ is the AWGN. The instantaneous SNR per received symbol is $\gamma=X^{2} E_{s} / N_{0}$, where $E_{s}=E\left\langle|s|^{2}\right\rangle$, with $|$.$| denoting$ absolute value and $N_{0}$ representing the single-sided power spectral density of the AWGN. The corresponding average SNR is $\bar{\gamma}=\Omega k E_{s} / N_{0}$. From [1] and by changing variables, the PDF of $\gamma$ can be obtained in a straight forward way as

$$
f_{\gamma}(\gamma)=\frac{2 \Xi^{(\beta+1) / 2} \gamma^{(\beta-1) / 2}}{\Gamma(m) \Gamma(k)} K_{\alpha}\left[2(\Xi \gamma)^{1 / 2}\right], \quad \gamma \geq 0
$$

where $\Xi=(k m) / \bar{\gamma}$.

The cumulative distribution function (CDF) of $\gamma$, can be derived by using [9, eq. (03.04.21.0007.01)] and (2), as

$$
\begin{aligned}
& F_{\gamma}(\gamma)=\pi \csc (\pi \alpha) \\
& \quad\left[\frac{(\Xi \gamma)^{m}{ }_{1} F_{2}(m ; 1-\alpha, 1+m ; \Xi \gamma)}{\Gamma(k) \Gamma(1-\alpha) \Gamma(1+m)}\right. \\
& \left.\quad-\frac{(\Xi \gamma)_{1} F_{2}(k ; 1+\alpha, 1+k ; \Xi \gamma)}{\Gamma(m) \Gamma(1+\alpha) \Gamma(1+k)}\right], \gamma \geq 0
\end{aligned}
$$

where ${ }_{p} F_{q}(\cdot)$ is the generalized hypergeometric function, [8, eq. (9.14/1)], and $p, q$ are integers.

The moments-generating function (MGF) is defined as $\mathcal{M}_{\gamma}(s) \triangleq \int_{0}^{\infty} \exp (-s \gamma) f_{\gamma}(\gamma) d \gamma$ [10]. By substituting (2) in this definition and by using [8, eq. (6.643/3)], the MGF of $\gamma$ can be expressed as

$$
\mathcal{M}_{\gamma}(s)=\left(\frac{\Xi}{s}\right)^{\beta / 2} \exp \left(\frac{\Xi}{2 s}\right) W_{-\beta / 2, \alpha / 2}\left(\frac{\Xi}{s}\right)
$$

where $W_{\lambda, \mu}(\cdot)$ is the Whittaker function, [8, eq. (9.220)].

Using (2) in the $n$th order moment, $\mu_{\gamma}(n)=E\left\langle\gamma^{n}\right\rangle$, an integral of the form $\mathcal{I}_{1}=\int_{0}^{\infty} \gamma^{(\beta-1) / 2+n} K_{\alpha}\left(2 \sqrt{\Xi} \gamma^{1 / 2}\right) d \gamma$ needs to be solved. By applying the transformation $t=\gamma^{1 / 2}$ in $\mathcal{I}_{1}$ and using [8, eq. (6.561/16)], the following expression is obtained

$$
\mu_{\gamma}(n)=\Xi^{-n} \frac{\Gamma(k+n) \Gamma(m+n)}{\Gamma(m) \Gamma(k)} .
$$

\section{B. Average Channel Capacity}

The average channel capacity, in the Shannon's sense is defined as $\bar{C} \triangleq \int_{0}^{\infty} B W \log _{2}(1+\gamma) f_{\gamma}(\gamma) d \gamma$, [11], where $B W$ is signal's transmission bandwidth. Hence, by substituting (2) in the above definition an integral of the form $\mathcal{I}_{2}=\int_{0}^{\infty} \gamma^{(\beta-1) / 2} \ln (1+\gamma) K_{\alpha}\left(2 \sqrt{\Xi} \gamma^{1 / 2}\right) d \gamma$ appears. By expressing $\ln (1+\gamma)$ as in [9, eq. (07.34.03.0456.01)] and $K_{\alpha}(\cdot)$ as in [9, eq. (03.04.26.0009.01)], this integral yields

$$
\mathcal{I}_{2}=\frac{1}{2} \int_{0}^{\infty} \gamma^{(\beta-1) / 2} G_{2,2}^{1,2}\left[\left.\gamma\right|_{1,0} ^{1,1}\right] G_{0,2}^{2,0}\left[\left.\Xi \gamma\right|_{\alpha / 2,-\alpha / 2} ^{-}\right] d \gamma
$$

where $G[\cdot]$ is the Meijer's $G$-function [8, eq. (9.301)]. Hence, $\mathcal{I}_{2}$ can be solved with the aid of [9, eq. (07.34.21.0011.01)] and $\bar{C}$ can be obtained in closed form as

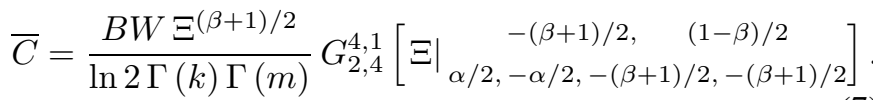

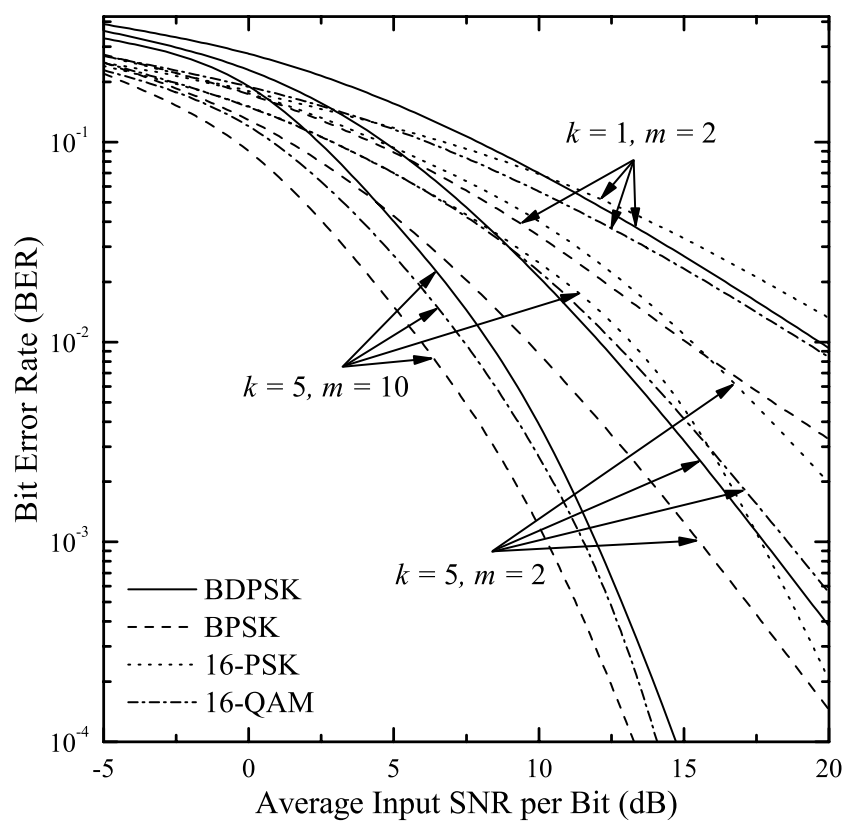

Fig. 1. BER of BDPSK, BPSK, Gray-encoded 16-PSK and 16-QAM signaling vs. $\bar{\gamma}_{b}$ for several values of $k$ and $m$.

\section{PERformance AnAlysis AND NumericAl Results}

In this section the performance of various classes of receivers operating over $K_{G}$ fading channels is presented in terms of BER $\left(\bar{P}_{b e}\right)$, outage probability $\left(P_{\text {out }}\right)$ and $\bar{C}$.

\section{A. Average Symbol Error Probability (SEP)}

The average SEP, $\bar{P}_{s e}$, can be evaluated directly by averaging the conditional symbol error probability, $P_{e}(\gamma)$, over the PDF of $\gamma$, i.e., $\bar{P}_{s e}=\int_{0}^{\infty} P_{e}(\gamma) f_{\gamma}(\gamma) d \gamma^{1}$. This yields:

- For BPSK and square M-QAM and for high values of the average input $\mathrm{SNR}, P_{e}(\gamma)=A \operatorname{erfc}(\sqrt{B \gamma})$, where $\operatorname{erfc}(\cdot)$ is the complementary error function [8, eq. (8.250/1)] and $A, B$ constants depending on the specific modulation scheme. By expressing $\operatorname{erfc}(\sqrt{B \gamma})=\sqrt{\pi}^{-1} G_{1,2}^{2,0}\left[\left.B \gamma\right|_{0,1 / 2} ^{1}\right]$ and using [9, eq. (07.34.21.0011.01)] the SEP can be obtained in closed form as

$$
\bar{P}_{s e}=\frac{A(\Xi / B)^{(k+m) / 2}}{\sqrt{\pi} \Gamma(m) \Gamma(k)} G_{2,3}^{2,2}\left[\frac{\Xi}{B} \mid \begin{array}{c}
(1-\beta) / 2,-\beta / 2 \\
\alpha / 2,-\alpha / 2,-(\beta+1) / 2
\end{array}\right] .
$$

- For non-coherent BFSK and BDPSK, $P_{e}(\gamma)=$ $A \exp (-B \gamma)$. As in obtaining (4), using again $[8$, eq. (6.643/3)], $\bar{P}_{\text {se }}$ can be expressed in closed form as

$$
\bar{P}_{s e}=A\left(\frac{\Xi}{B}\right)^{\beta / 2} \exp \left(\frac{\Xi}{2 B}\right) W_{-\beta / 2, \alpha / 2}\left(\frac{\Xi}{B}\right) .
$$

Note that for $m=1$ and BDPSK, i.e., $A=1 / 2, B=1$, by using [8, eq. (9.220)], [9, eq. (07.20.03.0020.01)] and [9, eq. (07.20.03.0002.01)], (9) reduces to a previously known expression [7, eq. (7)]. 


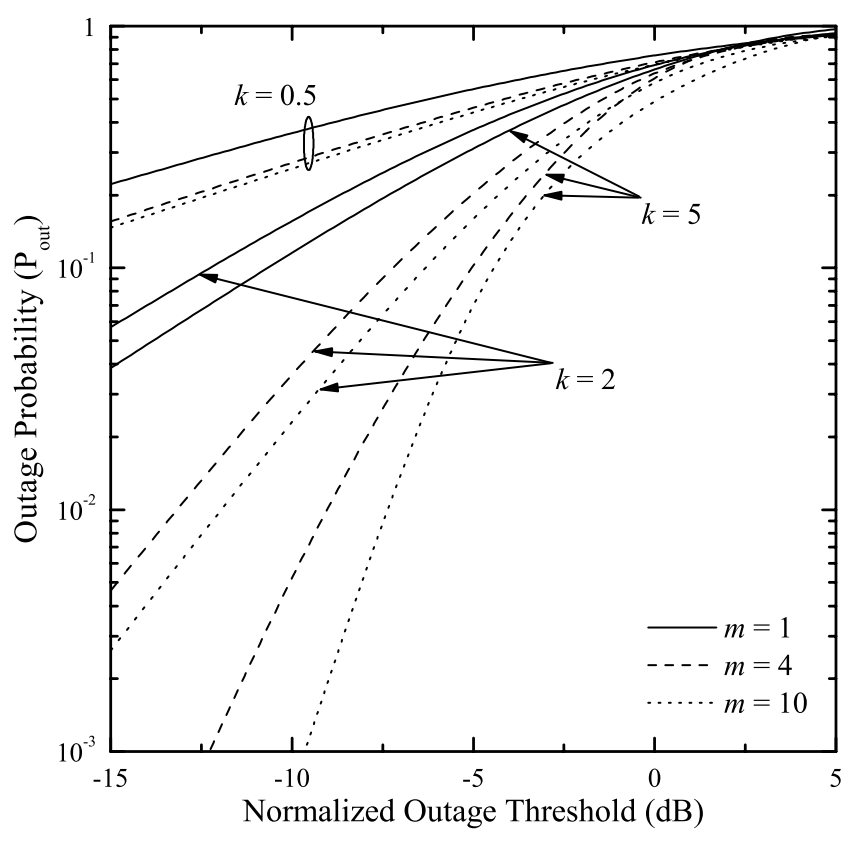

Fig. 2. $\quad P_{\text {out }}$ vs. $\gamma_{t h} / \bar{\gamma}_{b}$, for several values of $k$ and $m$.

- For Gray encoded $M$-PSK and $M$-DPSK, $P_{e}(\gamma)=$ $A \int_{0}^{\Lambda} \exp [-B(\theta) \gamma] d \theta$, where $\Lambda$ is constant. Thus, similarly to (9), the SEP can be expressed as

$$
\bar{P}_{s e}=A \Xi^{\beta / 2} \int_{0}^{\Lambda} \frac{\exp \left[\frac{\Xi}{2 B(\theta)}\right]}{B(\theta)^{\beta / 2}} W_{-\beta / 2, \alpha / 2}\left[\frac{\Xi}{B(\theta)}\right] d \theta \text {. }
$$

The above finite integral can be evaluated via numerical integration, using any of the well known mathematical software packages. In Fig. 1, the BER is plotted for BDPSK, BPSK, 16PSK and 16-QAM signaling with Gray encoding, as a function of the average input SNR per bit, $\bar{\gamma}_{b}=\bar{\gamma} / \log _{2} M$, for several values of $k$ and $m$. As expected, the BER improves as $\bar{\gamma}_{b}$ increases, while for a fixed value of $\bar{\gamma}_{b}$, BER also improves with an increase of $k$ and/or $m$.

\section{B. Outage Probability $\left(P_{\text {out }}\right)$}

Outage probability is defined as the probability that the received SNR falls below a given threshold, $\gamma_{\text {th }}$, and can be simply obtained as $P_{\text {out }}\left(\gamma_{\text {th }}\right)=F_{\gamma}\left(\gamma_{\text {th }}\right)$. Fig. 2 shows $P_{\text {out }}$ versus normalized $\gamma_{\mathrm{th}}, \gamma_{\mathrm{th}} / \bar{\gamma}_{b}$, for several values of $k$ and $m$. $P_{\text {out }}$ decreases (i.e., the outage performance improves) with an increase of $k$ and/or $m$. However the gap among the curves decreases as $k$ and/or $m$ increase.

\section{Average Channel Capacity $(\bar{C})$}

The normalized $\bar{C}$ can be easily obtained as $\bar{C} / B W$, in terms of b/s/Hz. In Fig. 3, $\bar{C} / B W$ is plotted as a function of $\bar{\gamma}_{b}$ for several values of $k$ and $m$. For comparison purposes, the normalized $\bar{C}$ for the AWGN and the Rayleigh channels are also plotted. As expected, $\bar{C}$ is always less than the capacity provided by the AWGN channel. Moreover, for $k=m=1$ the obtained average capacity is less than in a Rayleigh fading channel, while it improves as $k$ and/or $m$ increase.

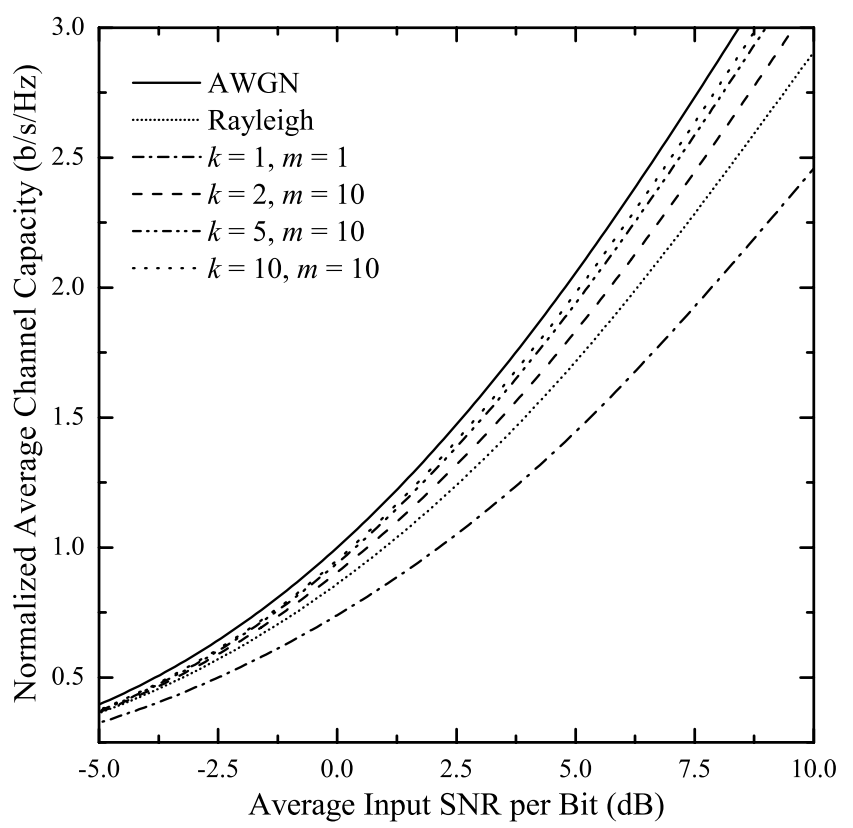

Fig. 3. $\bar{C} / B W$ vs. $\bar{\gamma}_{b}$, for several values of $k$ and $m$.

\section{ACKNOWLEDGMENTS}

This work has been performed within the framework of the Satellite Network of Excellence (SatNEx) project (IST507052), a Network of Excellence (NoE) funded by European Commission (EC) under the FP6 program.

\section{REFERENCES}

[1] M. K. Simon and M.-S. Alouini, Digital Communication over Fading Channels, 2nd ed. New York: Wiley, 2005.

[2] P. M. Shankar, "Error rates in generalized shadowed fading channels," Wireless Personal Communications, vol. 28, no. 4, pp. 233-238, Feb. 2004.

[3] D. R. Iskander and A. M. Zoubir, "Estimation of the parameters of the $K$-distribution using higher order and functional moments," IEEE Trans. Aerosp. Electron. Syst., vol. 35, no. 4, pp. 1453-1457, Nov. 1999.

[4] S. Chitroub, A. Houacine, and B. Sansal, "Statistical characterisation and modelling of SAR images," Elsevier, Signal Processing, vol. 82, no. 1, pp. 69-92, Jan. 2002.

[5] A. Abdi and M. Kaveh, " $K$ distribution: An appropriate substitute for Rayleigh-lognormal distribution in fading-shadowing wireless channels," Electron. Lett., vol. 34, no. 9, pp. 851-852, Apr. 1998.

[6] D. R. Iskander, A. M. Zoubir, and B. Boashash, "A method for estimating the parameters of the K-Distribution," IEEE Trans. Signal Processing, vol. 47, no. 4, pp. 1147-1151, Apr. 1999.

[7] A. Abdi and M. Kaveh, "Comparison of DPSK and MSK bit error rates for $K$ and Rayleigh-Lognormal fading distributions," IEEE Commun. Lett., vol. 4, no. 4, pp. 122-124, Apr. 2000.

[8] I. S. Gradshteyn and I. M. Ryzhik, Table of Integrals, Series, and Products, 6th ed. New York: Academic Press, 2000.

[9] "The Wolfram functions site." [Online]. Available: http://functions.wolfram.com

[10] A. Papoulis, Probability, Random Variables, and Stochastic Processes, 2nd ed. New York: McGraw-Hill, 1984.

[11] W. C. Y. Lee, "Estimate of channel capacity in Rayleigh fading environment," IEEE Trans. Veh. Technol., vol. 39, no. 3, pp. 187-189, Aug. 1990. 